\title{
Can Lokomat therapy with children and adolescents be improved? An adaptive clinical pilot trial comparing Guidance force, Path control, and FreeD
}

Tabea Aurich-Schuler ${ }^{1,2^{*}+}$, Fabienne Grob ${ }^{1,2,3+}$, Hubertus J.A. van Hedel ${ }^{1,2}$ and Rob Labruyère ${ }^{1,2}$

\begin{abstract}
Background: Robot-assisted gait therapy is increasingly being used in pediatric neurorehabilitation to complement conventional physical therapy. The robotic device applied in this study, the Lokomat (Hocoma AG, Switzerland), uses a position control mode (Guidance Force), where exact positions of the knee and hip joints throughout the gait cycle are stipulated. Such a mode has two disadvantages: Movement variability is restricted, and patients tend to walk passively. Kinematic variability and active participation, however, are crucial for motor learning. Recently, two new control modes were introduced. The Path Control mode allows the patient to walk within a virtual tunnel surrounding the ideal movement trajectory. The FreeD was developed to support weight shifting through mediolaterally moveable pelvis and leg cuffs. The aims of this study were twofold: 1) To present an overview of the currently available control modes of the Lokomat. 2) To evaluate if an increase in kinematic variability as provided by the new control modes influenced leg muscle activation patterns and intensity, as well as heart rate while walking in the Lokomat.
\end{abstract}

Methods: In 15 adolescents with neurological gait disorders who walked in the Lokomat, 3 conditions were compared: Guidance Force, Path Control, and FreeD. We analyzed surface electromyographic (sEMG) activity from 5 leg muscles of the more affected leg and heart rate. Muscle activation patterns were compared with norm curves.

Results: Several muscles, as well as heart rate, demonstrated tendencies towards a higher activation during conditions with more kinematic freedom. sEMG activation patterns of the M.rectus femoris and M.vastus medialis showed the highest similarity to over-ground walking under Path Control, whereas walking under FreeD led to unphysiological muscle activation in the tested sample.

Conclusions: Results indicate that especially Path Control seems promising for adolescent patients undergoing neurorehabilitation, as it increases proximal leg muscle activity while facilitating a physiological muscle activation. Therefore, this may be a solution to increase kinematic variability and patients' active participation in robot-assisted gait training.

Keywords: Youths, Cerebral Palsy, Neurological gait disorders, Robot-assisted gait therapy, Impedance control, FreeD motion, Surface Electromyography, Kinematic variability

\footnotetext{
* Correspondence: tabea.aurich@kispi.uzh.ch

${ }^{\dagger}$ Equal contributors

${ }^{1}$ Rehabilitation Center Affoltern am Albis, Children's University Hospital

Zurich, Muehlebergstrasse 104, CH-8910 Affoltern am Albis, Switzerland

${ }^{2}$ Children's Research Center, Children's University Hospital Zurich,

Steinwiesstrasse 75, CH-8032 Zurich, Switzerland

Full list of author information is available at the end of the article
} 


\section{Background and technical introduction}

Walking disorders are a common problem in patients with neurological impairments. Accordingly, robot-assisted therapy is used in neurorehabilitation to increase the dose of task-specific gait training. The most frequently applied gait orthosis is the Lokomat (Hocoma AG, Volketswil, Switzerland). The Lokomat is a robotic exoskeleton, used in rehabilitation centers to complement conventional therapies since it appears to be a feasible and promising therapeutic tool for adults as well as for children and adolescents [1-5]. Nevertheless, its effectiveness is being controversially discussed. Some studies concluded that robot-assisted therapy is superior to manual or conventional therapy in patients with stroke $[6,7]$ while other studies came to the opposite conclusion for stroke survivors as well as for patients with spinal cord injury [8-11]. Recent research, especially with patients following stroke, pointed out that a combination of robot-assisted therapy and conventional physical therapy might be the most promising solution $[1,3]$. Thereby, robotic devices could offer a safe, simplified, and supportive environment for the therapy while also supporting visual feedback and haptic learning, which is thought to lead to the best learning performance of movements $[12,13]$. However, clinical routine and scientific evidence showed that the provision of such a supportive environment comes with a price: the full and constant guidance of the robot often leads to patients being passive which might result in reduced muscle activity [14-16]. It furthermore limits active participation, dynamic walking pattern adaptation, variability in movements, and the possibility to make errors. These are all important factors for motor learning and for improving gait performance [16-20]. Patients have to train in many different ways and as often as possible ("repetition without repetition") [21]. In an animal study, Cai et al. [22] demonstrated that spinal cord-transected mice showed a faster and a more distinct recovery when they trained with variable compared to fixed robotic trajectories for movements of the hind limbs. Although these results are encouraging, it remains unclear if these findings also translate to humans. Anyhow, improvement of rehabilitation robots, especially for functionally more advanced patients, is essential.

Consequently, new approaches have been developed that take into account the patient's functional ability. These are based on the technical reduction of the supportive force to ensure active participation of the patient as well as to increase the possible variability of the movement [23]. The following paragraphs provide a comprehensive overview of the commercially available control soft- and hardware of the Lokomat.

\section{Control modes of the Lokomat}

Currently, two different commercially available modes exist to quantify and modify the amount of support the patients receive during walking: Guidance Force and Path Control.

\section{Guidance force}

The original mode Guidance Force can be set from 0 to $100 \%$. Walking at $100 \%$ guidance (impedance control) corresponds to a position-controlled mode, i.e. there is a predetermined cyclical movement trajectory for the knee and hip in the sagittal plane from which no deviation is possible [24-26]. Therefore, theoretically, no active participation of the patient is needed. From a clinical perspective, this might be a solution for severely affected patients [27].

As soon as the Guidance Force parameter is $<100 \%$, the impedance is reduced, which means that the restoring forces that push the patient's hip and knee towards the reference trajectory are reduced. Therefore, small deviations from the given trajectory are allowed, and the greater the deviation is, the larger becomes the force that pushes the patient back to the trajectory (like a spring). When Guidance Force is set to $0 \%$, the Lokomat will not provide support for the patient's movements and should only compensate for robotic dynamics (gravity and Coriolis forces) but not for inertia. The downside of robotic devices using classical impedance control is the temporal restriction in walking. They hinder the patients to vary their timing without losing control in space and to experience kinematic variability in a safe and supporting way [26].

\section{Path control}

The first solution to solve this issue with the limited variability in kinematics of exoskeleton robots was proposed by Cai et al. [28] in mice, and it was adapted to stroke patients by Banala et al. [29]. A virtual tunnel was implemented, in which the patients could modify their trajectories with a certain spatial and temporal freedom while a moving force supported them to conduct the movement in accordance with the treadmill speed. For the Lokomat, the first version of this strategy called Path Control was implemented by Duschau-Wicke et al. [26], and it has been commercially implemented in all Lokomat Pro version 6.0 devices since 2014. In Path Control, kinematic variability is offered by a torque field tunnel in joint space which controls the spatiotemporal characteristics of the gait pattern by applying corrective torques if the leg position is outside of the tunnel. The width of the tunnel can be set to narrow (small deviations allowed), middle, and wide (large deviations allowed). The "Support Force" (0-100\%) assists the patient with the step timing. It provides an extra "wind" of force in the direction of the gait trajectory and can help the patient temporally to overcome weakness. It also reduces the uncompensated inertia of the robot (Fig. 1, for 

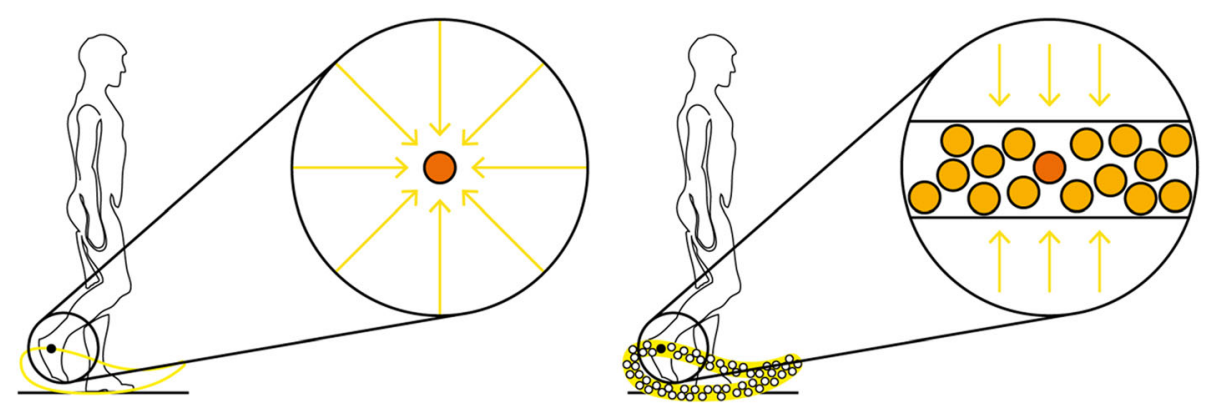

Fig. 1 Guidance Force and Path Control mode. Left: the Guidance Force mode. Right: Path Control mode. The orange dots indicate the required position in the sagittal plane at a specific time point in the gait cycle (spatiotemporal placement). The yellow arrows represent the forces that push the patient to the reference trajectory (Guidance Force) or the tunnel (Path Control). The additional dots on the right side indicate several possible positions, symbolizing the kinematic variability. Images courtesy of Hocoma AG

technical details, see [26, 30, 31]). Since Guidance Force and Path Control are superimposed mechanisms, the Guidance Force mode must be set to lower than $30 \%$ to enable Path Control to unfold its advantages.

Only a few studies have investigated Path Control in adult humans. They could show an increased active participation [30], a more physiological gait pattern [27], and improvements in clinical gait parameters when training with Path Control instead of Guidance Force $[14,32]$. So far, no study exists that implemented the Path Control mode in children and adolescents with neurological gait disorders.

\section{FreeD}

Up to this stage, the pelvis and its motions were constrained to the sagittal plane and also the leg cuffs were fixed preventing lateral movements during Lokomat walking. However, lateral pelvic displacement is physiological and necessary for a natural gait pattern [33]. Different studies with robot-assisted devices concluded that restrictions in pelvic motions severely affect gait dynamics [34] and alter muscle activation patterns $[8,15]$ and, therefore, should be avoided.

In October 2014, a new module for the Lokomat was introduced: The FreeD. With this hardware and software approach, the pelvis is now movable in the frontal plane to a lateral translation of up to $4 \mathrm{~cm}$ (per side) and in the transversal plane to a pelvic rotation of up to $4^{\circ}$ (per side). Additionally, the cuffs have a laterally movable range (Fig. 2). This should support the natural lateral pelvis displacement as well as weight shifting during walking and might enable an additional balance training [35] which would be useful since balance is often particularly affected in patients with neurological disorders [34]. Another study showed that with rhythmic weightshifting training, gait performance in children with spastic diplegic cerebral palsy could be increased [36]. Therefore, the FreeD might be a promising renewal for robot-assisted gait training, "making the walking pattern more physiological and more natural" [35].

Although a substantial amount of work has been done during the last years in this rapidly growing field, the question concerning the most effective control algorithm for robot-assisted gait training still remains open [23]. Therefore, the aim of this study was to investigate the new control modes Path Control and FreeD in children and adolescents with neuromotor disorders and to compare them to Guidance Force. To examine the influence of these control modes on sEMG parameters during Lokomat walking, we formulated the following research questions: (1) Quantitative gait analysis: Does training in the Lokomat under a condition with more kinematic freedom leads to an increase in muscle activity and heart rate (Guidance Force < Path Control < FreeD)? and (2) Qualitative gait analysis: Does a change in muscle activity go along with maintaining a physiological gait pattern?

\section{Methods}

\section{Participants}

Thirty-seven in- and out-patients of the Rehabilitation Center for Children and Adolescents in Affoltern am Albis were recruited between April 2015 and 2016 to join the study. They met the following inclusion criteria: (1) a neurological impairment resulting in a gait disorder, (2) no contraindications for the training in the Lokomat (see [37]), (3) able to communicate fear, discomfort or pain, and (4) understanding simple instructions, (5) a femur length of $35-47 \mathrm{~cm}$ (currently, Path Control is only available for the adult Lokomat orthosis) and (6) written informed consent of parents and adolescents $\geq 14$ years and assent of children $<14$ years. Sixteen children and adolescents agreed to participate in the study. Twenty-one disagreed due to different private reasons (e.g. distance for traveling to the clinic, busy at school). Patients were characterized by age, daily life mobility aids, and the Gross Motor Function Classification System (GMFCS level, only available for children 


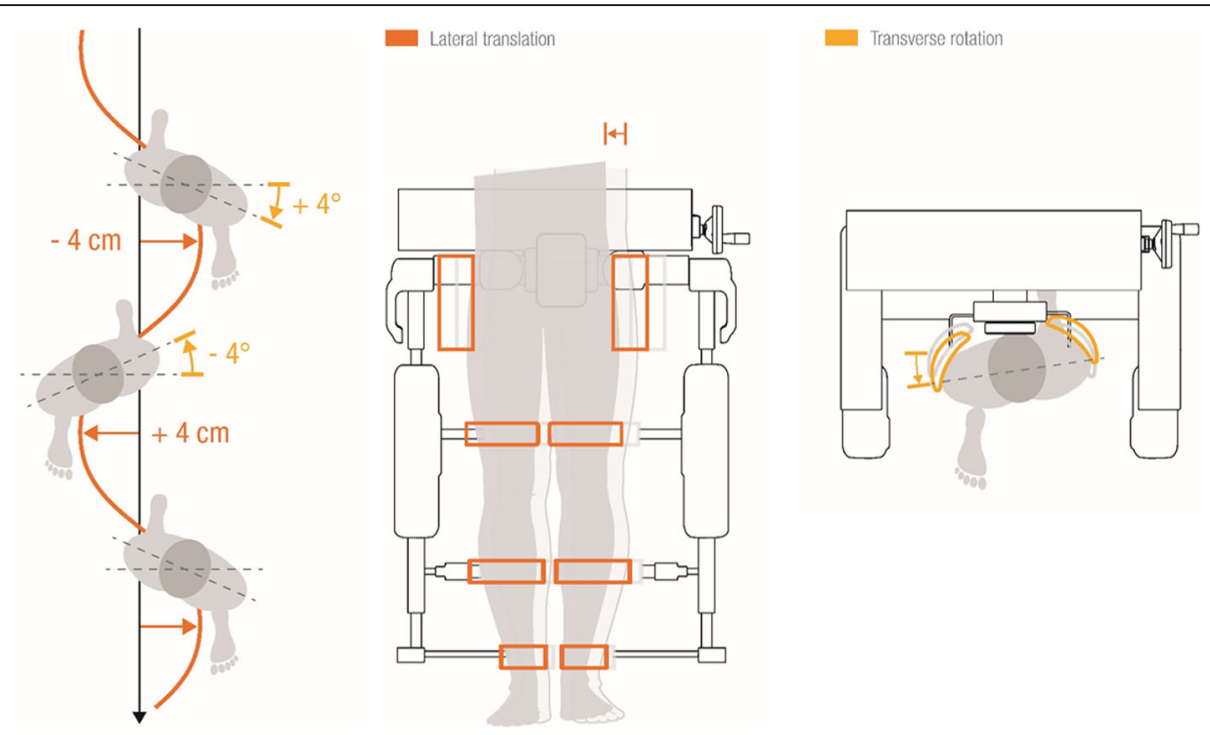

Fig. 2 Lateral translation and transverse rotation of FreeD. Left: Lateral pelvis movement and rotation during physiological walking. Middle: Possibility of lateral pelvis and leg translation with the new FreeD. Right: Possibility of pelvis rotation with the new FreeD. Images with courtesy of Hocoma $A G$

and adolescents with cerebral palsy [38]). Measurements of the Manual Muscle Test [39] and the Selective Control Assessment for the Lower Extremity [40] were performed to determine the more affected leg of the patients. The completed STROBE checklist (see Additional file 1) and source data (see Additional files 2, 3, 4, and 5) can be found in the appendix.

\section{Gait training robot and control modes}

Detailed information about the used Lokomat device can be found elsewhere [24, 41, 42]. In this study, the Lokomat exoskeleton was adapted to every patient individually, ensuring that walking in the robot was as comfortable as possible. Training parameters were selected by clinical experience [43]. The treadmill was set to a comfortable speed for the participant (initial speed was always $1.8 \mathrm{~km} / \mathrm{h}$ and participants could then change it in small steps until they found it to be comfortable). Average speed \pm standard deviation was $1.96 \pm 0.15 \mathrm{~km} / \mathrm{h}$ and it was kept constant during the measurements. The amount of body weight support was set to $30 \%$ of the child's body weight. Patients had to wear elastic foot lifters to support toe clearance during swing phase and a mirror provided visual feedback of the walking pattern. To allow for a warm-up (all participants had prior Lokomat experience), each patient walked about $10 \mathrm{~min}$ with 100\% Guidance Force (baseline walking setting). After this familiarization period, all patients reported that walking in the device felt comfortable and that the kinematic trajectory was easy to follow.

\section{Experimental design}

The exact device settings for the three experimental conditions are depicted in Fig. 3. The order of the conditions was randomized [44], and each condition lasted $2 \mathrm{~min}$. Data recording occurred during these $2 \mathrm{~min}$, and standardized instructions were given before and throughout the testing (see Additional file 7). Between the conditions, a break of 1 min with the baseline walking setting allowed the patient to relax for a moment.

As already mentioned in the technical background, Guidance Force and Path Control are superimposed

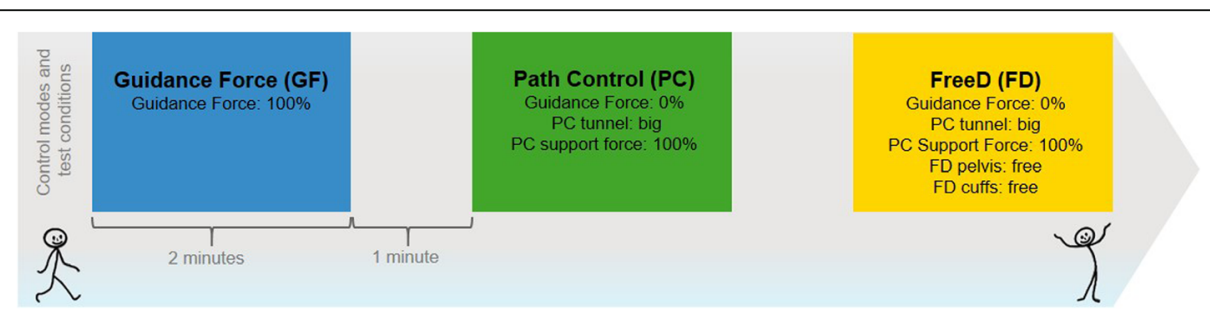

Fig. 3 Study overview. Overview of the three different control modes and test conditions. The order was randomized and every condition lasted two minutes with a one minute break in between 
control modes, which optionally can be combined with the FreeD module. Therefore, condition GF was a standard therapy setting of $100 \%$ Guidance Force (see blue box in Fig. 3). Condition PC was a setting with higher kinematic freedom (Path Control), whereby Guidance Force was set to $0 \%$ to allow Path Control to completely take over (see green box in Fig. 3). To max out the Lokomat's kinematic freedom, condition FD was a combination of Path Control and FreeD, whereby the pelvis and cuffs were moveable (pelvis free up to $4^{\circ}$ of rotation and $2 \mathrm{~cm}$ lateral shift to each side; cuffs free, see yellow box in Fig. 3). In both, Path Control and FreeD, the Support Force within Path Control was set to $100 \%$ (see green and yellow boxes in Fig. 3).

The study consisted of further sub-conditions where the Support Force during Path Control was modulated (comparable to [31]), and the degrees of freedom (pelvis and cuffs) of the FreeD were modified, but to remain concise, they are not part of this manuscript.

\section{Measurements}

The measurements took place at the Rehabilitation Center for Children and Adolescents in Affoltern am Albis, Switzerland. The experimental protocol was in accordance with the Declaration of Helsinki [45] and was approved by the Ethical Committee of the Canton Zurich, Switzerland. Activity of the following 5 muscles of the more affected leg was determined by surface electromyography (sEMG): the M.rectus femoris (RF), M.vastus medialis (VM), M.biceps femoris, long head (BF), M.tibialis anterior (TA) and M.gastrocnemius lateralis (GL). The surface electromyography recordings were done with the Wireless TeleMyo DTS system and the MyoResearch XP software (Noraxon Inc., Scottsdale, USA). The system was time-synchronized with a video camera that was positioned beside the child's measured leg to identify gait cycle events. The placement of the sEMG electrodes was always done by the same therapist for all measurements adhering as closely as possible to the SENIAM guidelines [46] (Fig. 4). The skin was prepared by shaving and applying an abrasive paste and then self-adhesive $\mathrm{Ag} / \mathrm{AgCl}$ snap electrodes (Noraxon Dual Electrodes, $10 \mathrm{~mm}$ diameter and $20 \mathrm{~mm}$ interelectrode distance, Noraxon Inc., Scottsdale, USA) were positioned. The quality of the sEMG signals was visually inspected during the familiarization period as well as during the measurements to ensure that the electrodes were correctly placed and to exclude movement artifacts during walking.

Furthermore, a heart rate belt (Polar RS800CX Pro Training, sampling frequency $0.2 \mathrm{~Hz}$, Polar Electro Oy, Kempele, Finland) recorded the heart rate during training. As the Guidance Force condition acted as theoretical baseline of physiological walking, we wanted to know if participants could maintain this physiological

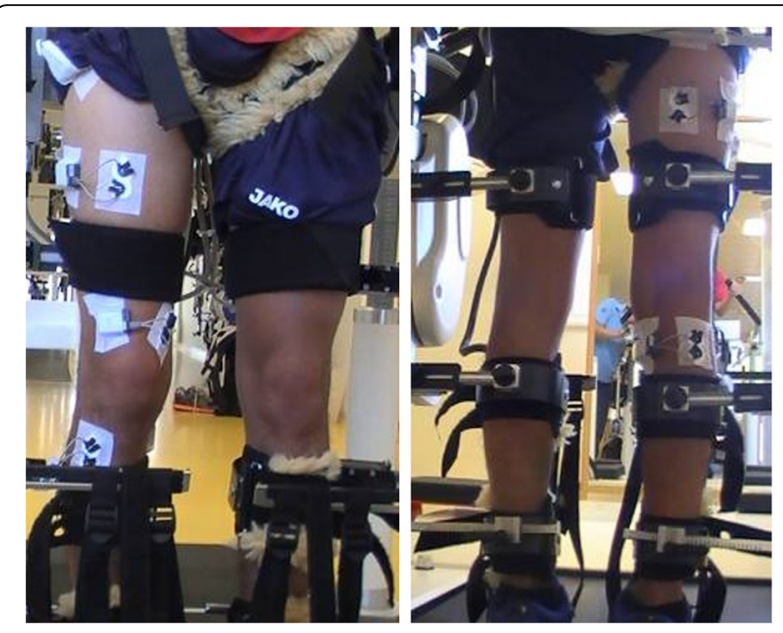

Fig. 4 sEMG electrodes placement. The placement of the electrodes (according to the SENIAM guidelines, [46]). Left (from top to bottom): M.rectus femoris, M.vastus medialis, M.tibialis anterior. Right (from top to bottom): M.biceps femoris long head, M.gastrocnemius lateralis

pattern when switching to other control strategies. Therefore, after each walking condition (see Fig. 3), the therapist scored the walking pattern as "physiological" $(=1)$ or "not physiological" $(=0)$. This decision was subjective, but it was mainly based on the fulfillment of the following factors: timing of heel strike, knee extension in stance phase, step symmetry, step length, toe clearance, and rhythm. If two or more factors were conspicuous, the performance was rated as "not physiological". Additionally, the patient scored the experience as "comfortable" $(=1)$ or "not comfortable" $(=0)$, as child-friendly terms for "physiological" and "not physiological". This was differentiated from signs or statements of physical discomfort or even pain, which led to a temporary halt of the experiment.

\section{Data analysis}

sEMG data were rectified and smoothed by a Root Mean Square with a time window of $100 \mathrm{~ms}$. For the sEMGdata analysis, 10 strides [47] after 30s of the start of each condition were analyzed. The markers for heel strike and toe off were set automatically by the program. Every single marker was controlled and checked visually with the synchronized video recording and adjusted manually if necessary. Afterward, the sEMG data were exported to Matlab (Matlab 7.1, the MathWorks Inc., Natick MA, USA). For the analysis investigating quantitative changes in sEMG activity, the sEMG of the 10 stance- and swing phases of each muscle for every condition of each patient were averaged. Then, they were merged and timenormalized to a $100 \%$ gait cycle (1000 samples).

For the analysis investigating qualitative changes in sEMG activity, we took the sEMG individual time- 
normalized averaged profile of each muscle for every condition. These gait curves were then amplitude-normalized to its maximal value (max. value $=100 \%$ ). Afterwards, we took the mean gait cycle over all participants per condition to generate grand-averaged gait cycle profiles for each muscle per condition. Based on these grand averages, we determined "On-"and "Off-phases" for each muscle. A muscle was considered to be 'on' if its activity exceeded a threshold, which was set at the minimum amplitude of the sEMG grand average per muscle plus two standard deviations (SD) (adapted from [48]). Then, these on- and off-phases were compared to the on- and off-phases of sEMG norm curve data from 87 typically developing children from Chang et al. [48], digitized with the WebPlotDigitizer (retrieved from [49]). Comparisons were made with two metrics: (i) the Spearman correlation coefficient that indicates the similarity with the norm curve; and (ii) the percentage of overlap as an indicator for the "normality" of the muscle activation pattern. It was calculated as: correct ON overlap $[\%]+$ correct OFF overlap correct ON overlap $[\%]+$ false ON overlap [\%]+correct OFF overlap $[\%]+$ false OFF overlap $[\%$

For the heart rate, the values for the 2 min were averaged for each condition.

\section{Statistics}

The statistics were done with IBM SPSS Statistics 22 (IBM Corporation, Armonk, NY, USA). Data were checked for normal distribution with the Shapiro-Wilk test together with Q-Q-plots and histograms. Because most of the data were not normally distributed, subsequent qualitative and quantitative analyses were done with non-parametric tests (Spearman correlations, Friedman- and Wilcoxon-tests). The significance level was set at $\alpha=5 \%$. Post-hoc corrections for multiple testing were done by applying False Discovery Rate corrected $p$-values (FDR, [50]). Additionally, effect sizes were calculated and scored according to Cohen's benchmarks ( $d=0.2$ is small, $d=0.5$ is medium, and $d=0.8$ is considered a large effect size, [51]). The correlations of the sEMG comparisons were interpreted as follows (adopted from Evans, [52]): $r<0.20$, "very weak"; 0.20-0.39, "weak"; 0.40-0.59, "moderate"; 0.60-0.79, "strong" and 0.80-1.00 "very strong relationship".

\section{Results}

Fifteen patients ( 5 girls, 10 boys) with a mean age of $16 \pm 2 y$ completed the trial. Details about the patients' characteristics are listed in Table 1. In one participant, the measurements had to be stopped immediately after the beginning for safety reasons (patient ID 13), as the therapist noted during the robot-walking familiarization period that the patient would not be able to walk with less than 100\% Guidance Force.

Since some of the participants had to wear lower leg orthoses for ankle-stabilization, we had missing data of the M.tibialis anterior for 2 participants and the M.gastrocnemius lateralis for 3 participants, respectively.

\section{Quantitative changes in sEMG activity and heart rate}

We found an increase in quantitative muscle activation in several muscles when the kinematic freedom of the Lokomat was enlarged. Significant group differences between the three conditions could be found for the M.rectus femoris $(P=0.038)$, the M.vastus medialis $(P=0.004)$ and the M.tibialis anterior $(P=0.018)$. Heart rate did not reach significant results but a trend could be detected $(P=0.085)$. sEMG amplitudes, the heart rate, and the $p$-values of the 3 conditions and the five muscles are presented in Fig. 5.

Additional file 7: Fig. S1 shows that the chosen protocol was adequately timed to approximately allow heart rate to reach a steady state during the conditions and return to baseline during the breaks.

Significant differences in quantitative muscle activation were found for M.rectus femoris, M.vastus medialis and M.tibialis anterior. Pairwise post-hoc comparisons with effect sizes of the different conditions are presented in Table 2.

Qualitative changes in SEMG activity and walking patterns The grand-averaged gait cycle profiles for each muscle and each condition are displayed in Fig. 6 together with reference curves of normally developing children adapted from Chang et al. [48].

Focussing on the therapist's and patient's scorings, the following results could be observed: During the Guidance Force condition, the therapist scored the gait pattern as "physiological" for all 15 patients while 13 patients rated the condition to be "comfortable" (15/13). These numbers were 8/9 during Path Control and 7/10 during FreeD motion, respectively. Apparently, all of the patients walked physiologically during the Guidance Force condition, whereas fewer patients were scored with a physiological gait pattern during Path Control and FreeD conditions. Table 3 summarizes the correlations and overlaps of the sEMG patterns with the norm curves and presents the therapist's and patients' scorings.

\section{Discussion}

\section{Quantitative changes in sEMG activity and heart rate}

Our study investigated changes in muscle activity levels and patterns induced by two new hard-/software approaches which were developed to increase patients' kinematic variability. In general, we could show partially significant increases in muscle activity and heart rate from the condition Guidance Force to Path Control (except for M.gastrocnemius lateralis) and from Path Control to FreeD motion (except for M.rectus femoris and heart rate) (Fig. 5 and Table 2). This is in line with 
Table 1 Patients' characteristics

\begin{tabular}{|c|c|c|c|c|c|}
\hline$\overline{\mathrm{ID}}$ & Age (years) & Main diagnosis (GMFCS Level) & More impaired leg & Walking speed $(\mathrm{km} / \mathrm{h})$ & Daily life mobility aids \\
\hline 1 & 19 & $\mathrm{CP}$, bilateral ataxic (III) & right & 2.2 & Dorsal walking frame for longer distances \\
\hline 2 & 19 & Hereditary spastic paraplegia & right & 2.2 & None \\
\hline 3 & 14 & $\mathrm{CP}$, bilateral ataxic (II) & left & 2.0 & Dorsal walking frame, ankle-foot orthoses \\
\hline 4 & 14 & $\mathrm{ABI}{ }^{\mathrm{a}}$ (unilateral paresis) & left & 1.9 & Foot-up orthosis \\
\hline 5 & 16 & $\mathrm{AB|}{ }^{a}$ (unilateral spastic paresis) & left & 2.0 & None \\
\hline 6 & 19 & $\mathrm{CP}$, bilateral spastic (III) & right & 2.0 & Crutches, orthopedic shoes \\
\hline 7 & 13 & $\mathrm{CP}$, bilateral spastic (II) & left & 2.0 & Foot-up orthosis \\
\hline 8 & 15 & MMC L3/L4 & right & 1.8 & Crutches \\
\hline 9 & 16 & $\mathrm{CP}$, bilateral spastic (II) & right & 1.8 & Ankle-foot orthoses \\
\hline 10 & 16 & CP, bilateral spastic (III) & left & 1.9 & $\begin{array}{l}\text { Dorsal walking frame for longer distances, } \\
\text { ankle-foot orthoses }\end{array}$ \\
\hline 11 & 14 & $\mathrm{CP}$, bilateral spastic (III) & right & 2.1 & Ankle-foot orthoses \\
\hline 12 & 20 & $\mathrm{ABI}{ }^{a}$ (bilateral spastic paresis) & right & 1.8 & None \\
\hline 13 & 19 & $\mathrm{CP}^{\mathrm{C}}$, bilateral spastic (IV) & right & 1.4 & Wheelchair, ankle-foot orthoses \\
\hline \multicolumn{6}{|c|}{ DROP-OUT } \\
\hline 14 & 15 & $\mathrm{CP}$, unilateral spastic (I) & left & 1.8 & None \\
\hline 15 & 14 & $\mathrm{ABI}{ }^{\mathrm{a}}$ (unilateral spastic paresis) & left & 1.8 & None \\
\hline 16 & 12 & $\mathrm{ABI}{ }^{\mathrm{b}}$ (unilateral paresis) & left & 2.1 & None \\
\hline
\end{tabular}

Abbreviations: CP cerebral palsy, GMFCS Gross Motor Function Classification System [38], MMC Meningomyelocele (spina bifida), ABI acquired brain injury, ${ }^{a}=$ measurements more than 2.5 years after event, ${ }^{b}=$ measurements 2 months after event. ${ }^{\mathrm{C}} \mathrm{ID} 13$ was excluded because the measurements had to be stopped shortly after the beginning

earlier results from Duschau-Wicke et al. [30] and Schück et al. [27], where patients walked more actively under the Path Control mode. However, in contrast to the results from Schück et al. [27], our results could detect only partially significant trends. This distinction could be explained by the different patient population or by the fact that their controls walked on a treadmill whereas we compared our data with norm curves of free overground walking. Another reason might be that we evaluated only one training session whereas they analyzed the change over

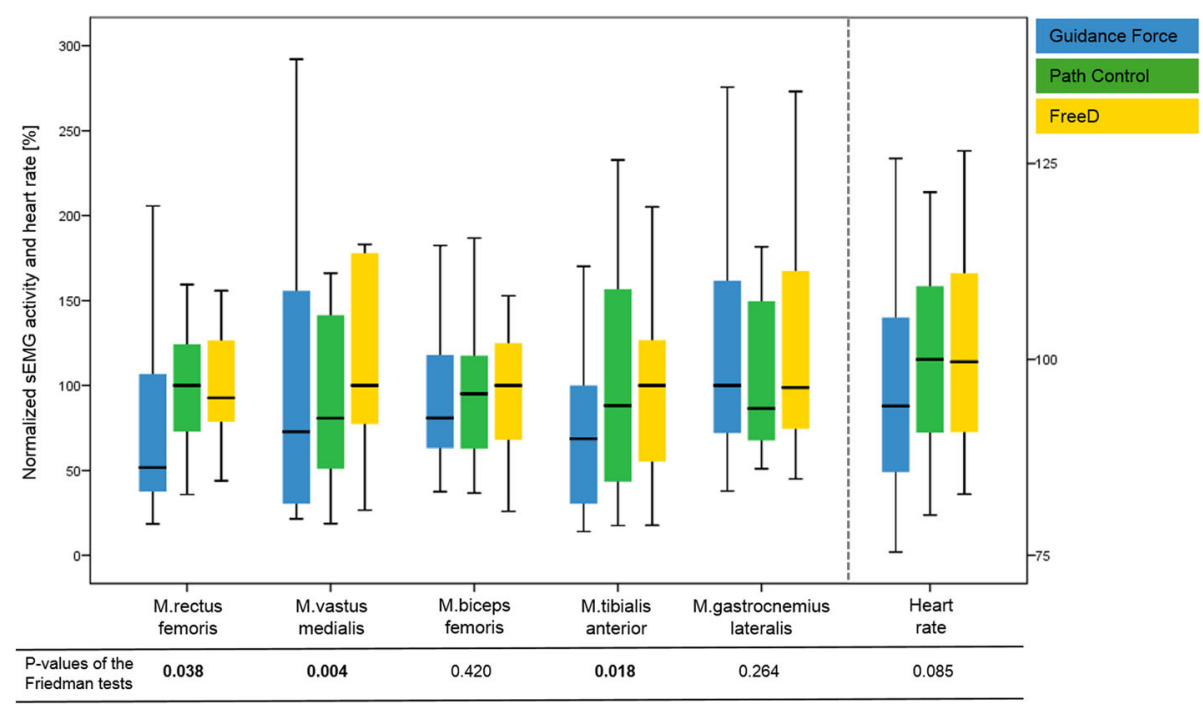

Fig. 5 sEMG amplitudes and heart rate during walking under the three different control modes. To facilitate a comparison, the conditions were normalized by setting the highest median sEMG activity value of the three walking conditions for each muscle to 100\% (and the same for heart rate). To improve visualization, outliers are not shown in the figure. However, they are included in the statistical analyses. P-values of the Friedman tests for each muscle and for heart rate are shown below the graph. Statistically significant data are indicated in bold 
Table 2 Comparison of the sEMG amplitudes between conditions. P-values of the Wilcoxon tests before and after FDR correction for multiple testing and effect sizes are shown

\begin{tabular}{|c|c|c|c|c|}
\hline & Comparisons & $p$-values of the Wilcoxon test & FDR corrected $p$-values & Effect sizes \\
\hline \multirow[t]{3}{*}{ M.rectus femoris } & GF - PC & 0.100 & 0.150 & -0.30 \\
\hline & GF - FreeD & 0.061 & 0.150 & -0.34 \\
\hline & PC - FreeD & 0.496 & 0.496 & -0.13 \\
\hline \multirow[t]{3}{*}{ M.vastus medialis } & $G F-P C$ & 0.173 & 0.173 & -0.25 \\
\hline & GF - FreeD & 0.020 & 0.030 & -0.43 \\
\hline & PC - FreeD & 0.002 & 0.006 & -0.56 \\
\hline \multirow[t]{3}{*}{ M.biceps femoris } & $G F-P C$ & 0.955 & 0.955 & -0.01 \\
\hline & GF - FreeD & 0.363 & 0.545 & -0.17 \\
\hline & PC - FreeD & 0.125 & 0.375 & -0.28 \\
\hline \multirow[t]{3}{*}{ M.tibialis anterior } & $G F-P C$ & 0.133 & 0.199 & -0.30 \\
\hline & GF - FreeD & 0.023 & 0.069 & -0.45 \\
\hline & $P C$ - FreeD & 0.701 & 0.701 & -0.08 \\
\hline \multirow[t]{3}{*}{ M.gastrocnemius lateralis } & $G F-P C$ & 0.209 & 0.582 & -0.26 \\
\hline & GF - FreeD & 1.000 & 1.000 & 0.00 \\
\hline & $P C$ - FreeD & 0.388 & 0.582 & -0.18 \\
\hline \multirow[t]{3}{*}{ Heart rate } & $G F-P C$ & 0.069 & 0.104 & -0.33 \\
\hline & GF - FreeD & 0.047 & 0.104 & -0.36 \\
\hline & PC - FreeD & 0.427 & 0.427 & -0.15 \\
\hline
\end{tabular}

Abbreviations: FDR False Discovery Rate [50], GF Guidance Force, $P C$ Path Control. Statistically significant data are indicated in bold

16 training sessions. Furthermore, van Kammen et al. [53] concluded that a higher guidance force in the Lokomat in general reduced the amplitude of muscle activity (M.biceps femoris, M.gastrocnemius medialis), and that this effect depended on body weight support and gait speed. However, they observed this effect only in muscles related to stability and propulsion and not leg loading (M.vastus lateralis) and foot clearance (M.tibialis anterior). In general, only small effect sizes could be found in the analysis of quantitative changes in sEMG and heart rate, except for the M.vastus medialis, where the significant differences in muscle activation were supported by a moderate effect size (i.e. -0.56, Table 2). It should be mentioned that the economics of a neurologically impaired gait (especially in cerebral palsy) are often affected by a poor biomechanical alignment. This means that a patient with cerebral palsy commonly walks with a high sEMG activity and heart rate. Therefore, a higher sEMG activity should not be a general goal of a therapy. Rather, it must be interpreted in combination with a pattern analysis.

Qualitative changes in SEMG activity and walking patterns A deeper insight in the sEMG grand-averaged gait cycle profiles (Fig. 6) revealed that the interpretation of the results is not as simple as it seems. The normal activity of M.rectus femoris (Fig. 6a) has two active phases, one at the end of preswing till midswing (55-80\% Gait Cycle (GC) [48]) and the other from terminal swing till the end of loading response (90-15\% GC) with a clear peak in loading response. In our study, walking under Path Control revealed the best overlap (70\%) with these active and passive phases as well as a moderate correlation $(r=0.57)$ with the pattern. This finding stands in contrast to Duschau-Wicke et al. [54] where M.rectus femoris presented an unphysiological gait pattern during walking with Path Control.

The M.vastus medialis normally activates in terminal swing till early midstance (86-20\% GC, Fig. 6b). At first sight, the pattern during walking under Guidance Force in our results looks the most similar to the norm curve, but it shows an abnormal active peak in late midstance. This is in contrast to the percentage overlap, which was best for the Path Control condition, i.e. 70\%. This high number has originated from the overlap of an active phase in initial contact and loading response (early stance phase) and a passive phase from midstance to the end of midswing. It might be confusing that the norm curve maps an additional preswing and initial swing activity (45-65\% GC, * in Fig. 6b), which is being ignored in the physiological activity range determined by Chang et al. [48]. This additional activity (in this study during walking under Guidance Force and FreeD) might stem from a co-contraction for the stabilization of the knee before entering the swing phase and was also reported in previous studies $[55,56]$. If this additional peak had been included by Chang et al. [48], walking under 

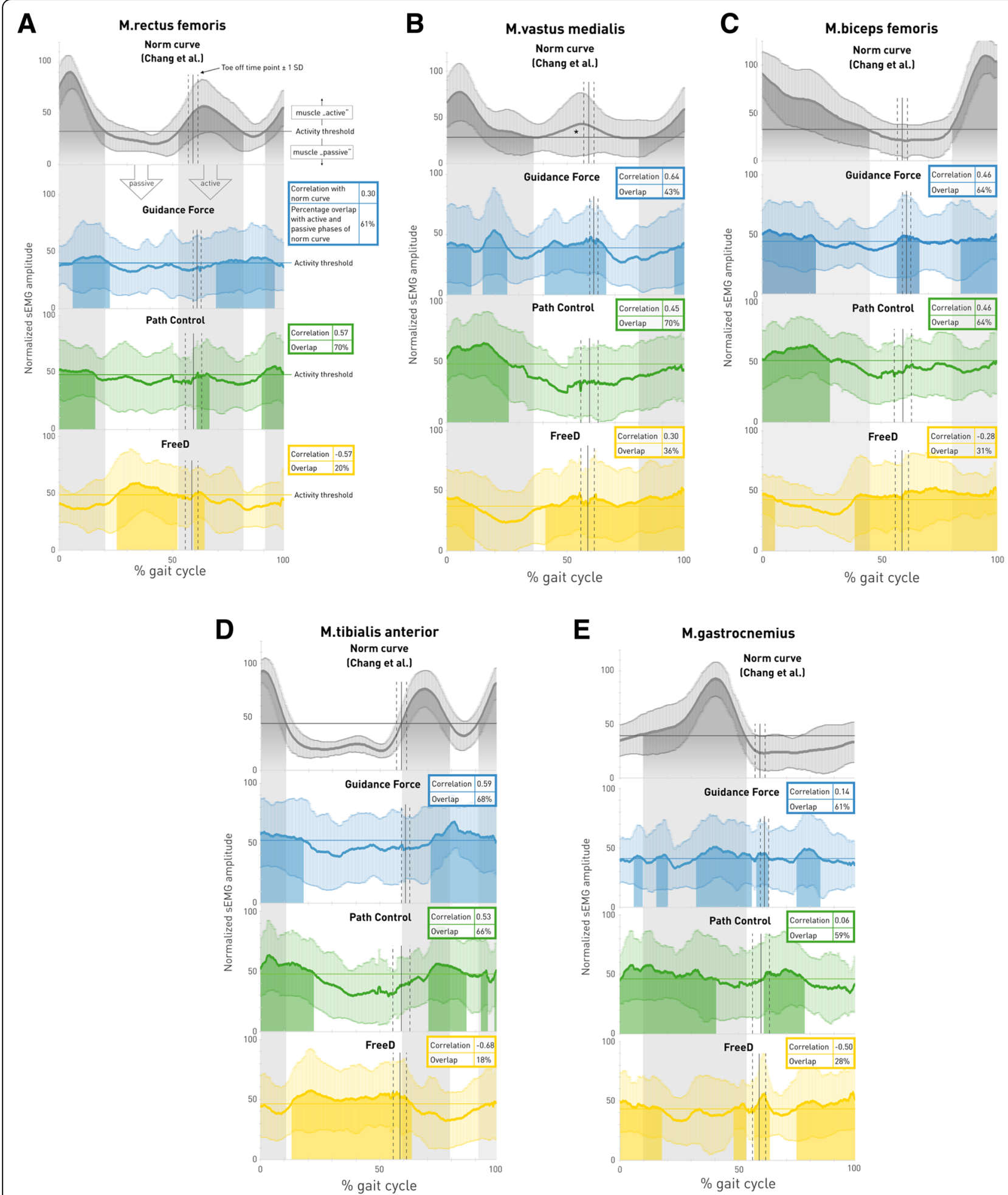

Fig. 6 (See legend on next page.) 
(See figure on previous page.)

Fig. 6 Grand-averaged gait cycle sEMG profiles for each muscle and each condition. Linear envelope curves of the averaged gait cycle per muscle show mean \pm standard deviation of the norm curve (adapted from Chang et al. [48]) and the three different walking conditions: Guidance Force (blue), Path Control (green) and FreeD (yellow). Muscle onset threshold was defined as 2 standard deviations above the minimum amplitude of the mean curve over all patients for each muscle separately. Grey banners in the background indicate that the muscle is expected to be "active" (= norm curve activity above the threshold); white banners in the background indicate that the muscle is expected to be "passive" (= norm curve activity below the threshold), see Fig. 6a. Colored shadows indicate that the muscle during that timepoint in the specific walking condition is active. Toe off time and shift from stance to swing phase is indicated with a vertical line \pm one standard deviation (dashed lines). The "correlation" value refers to the Spearman correlation of the pattern of that specific walking condition with that of the norm pattern and "overlap" indicates the percentage of "activity" and "passivity" overlap of the pattern of that specific walking condition with that of the norm curve. *According to Chang et al. [48], the M.vastus medialis is not active here, despite supra-threshold activity

Guidance Force would have gained the best agreement between the two patterns (which is already visible with the current strong correlation $r=0.64$ ).

The active range of M.biceps femoris is from the end of midswing till terminal stance (80-50\% GC, Fig. 6c). In our study, this was best represented by both Guidance Force and Path Control with a moderate correlation $(r=0.46)$ and an overlap of $64 \%$.

M.tibialis anterior is characterized by two peaks of activity, one from preswing till midswing (55-80\% GC) and a second in terminal swing until the end of loading response $(90-15 \%$ GC, Fig. 6d). In this muscle, both walking under Guidance Force as well as under Path Control could reach an acceptable overlap (68\% and 66\%, respectively), with moderate correlations. An abnormally low sEMG activity in terminal swing as detected in our data was also reported in other studies with children $[56,57]$ and could stem from the use of foot lifters [15].

The M.gastrocnemius lateralis normally presents activity from the end of the loading response to the middle of preswing (10-55\% GC), with a clear peak at $40 \% \mathrm{GC}$ (Fig. 6e). Our data could not detect this clear pattern with only very weak correlations during Guidance Force $(r=0.14)$ and Path Control $(r=0.06)$. Main functions of this muscle are to extend and stabilize the knee during stance and to induce the push off. When walking in the Lokomat, the cuffs and the robot itself take over these parts. A pattern without clear activity peaks of the M.gastrocnemius is also known from treadmill walking, where the push off is not as clear due to the moving treadmill [56]. An early onset of swing and a prolonged activity in stance are known as the plantar flexion-knee extension couple to control the second rocker and an

Table 3 Overview of the sEMG correlations and overlaps with the norm curves and subjective ratings of the therapist and patients

\begin{tabular}{l|c|c|c|c|c|c}
\hline & \multicolumn{2}{|c|}{ Guidance Force } & \multicolumn{2}{c|}{ Path Control } & \multicolumn{2}{c}{ FreeD } \\
\cline { 2 - 7 } & Correlation & Overlap & Correlation & Overlap & Correlation & Overlap \\
\hline M.rectus femoris & 0.30 & $61 \%$ & 0.57 & $70 \%$ & -0.57 & $20 \%$ \\
\hline M.vastus medialis & 0.64 & $43 \%$ & 0.45 & $70 \%$ & 0.30 & $36 \%$ \\
\hline M.biceps femoris & 0.46 & $64 \%$ & 0.46 & $64 \%$ & -0.28 & $31 \%$ \\
\hline M.tibialis anterior & 0.59 & $68 \%$ & 0.53 & $66 \%$ & -0.68 & $18 \%$ \\
\hline $\begin{array}{l}\text { M.gastrocnemius } \\
\text { lateralis }\end{array}$ & 0.14 & $61 \%$ & 0.06 & $59 \%$ & -0.50 & $28 \%$ \\
\hline
\end{tabular}

\begin{tabular}{l|c|c|c}
\hline $\begin{array}{l}\text { Sum of therapist's } \\
\text { rating }\end{array}$ & 15 & 8 & 7 \\
\hline $\begin{array}{l}\text { Sum of patient's } \\
\text { rating }\end{array}$ & 13 & 9 & 10 \\
\hline
\end{tabular}

Therapists had to score the patient's walking under a specific condition as "physiological" (=1) or "not physiological" (=0); Patients had to score the walking under a specific condition as "comfortable" $(=1)$ or "not comfortable" $(=0)$. The color codes are adapted in accordance to the interpretation for the correlations [52]: "very weak" (or negative) and "weak" = white, "moderate" = light grey and "strong" = dark grey 
upright position [56]. In our data, this was observable in Path Control and FreeD conditions.

In contrast to the norm data, our pattern curves lack clearly discernible peaks. At this point, it must be mentioned that Chang et al. [48] generated their norm curves by including only EMG patterns which showed the typical one or two peaks in the expected period (positive selection bias). It is obvious that the elimination of all other patterns resulted in curves with clear peaks and clear active and passive phases. In contrast, our grand-average profiles included all data. Therefore, it is difficult to get good correlations with these norm patterns, and we decided to add the overlap analysis. Thereby, it is possible to observe windows of abnormal activity, similarly to the work of van Kammen et al. [53]. This abnormal activity may indicate efforts e.g. to overcome constraints from the robot $[15,16]$ or increased balance needs $[53,58]$.

In summary, proximal leg muscle activity patterns did have the best overlaps during walking under Path Control, with also good patterns under Guidance Force. Distal leg muscles showed conflicting outcomes. While M.tibialis anterior generated quite good patterns concerning correlation and overlap, weaker correlations were found for M.gastrocnemius lateralis. Generally, during the FreeD motion condition, all pattern correlations were weak and mostly negative. However, FreeD was the only condition, where significantly higher muscle activity was found in the M.vastus medialis compared to Guidance Force or Path Control. The higher muscle activation could be a result of the efforts of the patients, who tried to compensate (unphysiologically) for too much kinematic freedom. Due to this kinematic freedom, we initially assumed that FreeD could facilitate physiological walking since patients would not be restricted in their gait trajectory. Hidler and Wall [15] indicated in their study that muscle activity patterns are altered while walking under full guidance because patients work against the robot (M.rectus femoris, M.biceps femoris). According to Ayoagi et al. [59], it is important for a natural human gait that robot-assisted devices allow the leg to swing out to the parasagittal plane and to allow the pelvis to rotate and to make a lateral translation. However, it seems that patients were unable to deal with the high kinematic freedom and therefore performed with too much muscular activity in an unnatural activation pattern to keep up the biomechanical alignment. It seems that this possibility to move to the parasagittal plane was the biggest challenge for our patients since the Guidance Force and Support Force vectors only operate in the sagittal plane.

Consequently, not only the sEMG analysis revealed issues with the kinematic freedom. Based on the therapist's rating we saw that not all of our patients could deal with the additional kinematic freedom during Path Control and FreeD and around half of the patients rated walking in these conditions as "uncomfortable". They developed an unphysiological gait pattern and would have needed more guidance from the Lokomat, or from the therapist through instructions. Due to the standardized instructions during the study (see Additional file 6), we refrained from this permanent verbal input. Nevertheless, these results were unanticipated since patients still received 100\% Support Force while walking with Path Control and FreeD. Moreover, patients reported very differently about their impression of the kinematic variability. While some of them found the freedom comfortable, others felt unguided and actively tried to deal with this variability. It seems clear that these feelings depend on the skills of the individual patient and these results are in line with the findings of Duschau-Wicke et al. [30]. Therefore, a further analysis of varying Support Forces may give additional information about the effects on muscle activity, activation patterns, and the rating of therapists and patients.

\section{Clinical implications}

For clinical practice in neurorehabilitation, it is important to train restorative rather than compensatory patterns [56, 60]. Therefore, inducing physiological gait patterns by training under Guidance Force or Path Control might be the preferred method. Nevertheless, our results reinforce the opinion that the therapist plays a crucial role during robot-assisted gait training during rehabilitation $[43,56]$. The therapist has to decide individually which control mode is reasonable for a training with a specific patient. Certainly, this decision depends also on the aim of the training. If the goal is to train a specific, symmetric gait pattern (often the target in an early phase of rehabilitation or in severely impaired patients) then the training should be performed using the Guidance Force mode where the patient gets an exact and symmetric, predetermined gait trajectory. If the goal is for example to train muscle strength, endurance, joint control, weight shifting, balance, or step variability, then Path Control or even FreeD might be a good choice. The therapist's decision to choose one or the other training option also depends on the duration with which a specific control mode is applied. A control mode with high kinematic freedom might be selected to work on a specific impairment, however, it will be difficult for the patient to train with a physiological walking pattern for a prolonged duration. In this case, the patient should get frequent, intensive verbal instructions.

\section{Limitations}

Our patient group was a convenience sample and heterogeneous regarding diagnosis, age, level of cognition, and skills. This may have increased the data variability 
and influenced the results, but the aim of this study was to reflect clinical everyday life with neurological patients where the population is very heterogeneous. Since the sample size in this study is small, we should be cautious in generalizing the results of this study to similar population groups, even though the uniformity of Lokomat therapy and the standardized measurement procedure provide a highly reproducible setting. Accordingly, further studies are needed to investigate disease-specific patterns into further detail to get a finegrained impression about the effects of the different control modes on the muscle activation patterns of patients with specific neuromotor disorders.

We compared sEMG-patterns of patients with neuromotor disorders to those of a published sample of healthy controls. While an intra-individual comparison would be desirable, these patients are not able to generate a physiological gait pattern overground. Therefore, our EMG data might partially differ from the EMG norm data, e.g. regarding exact electrode placement or data preprocessing. Additionally, we cannot exclude that the $10 \mathrm{~min}$-long warm-up phase with $100 \%$ Guidance Force influenced the performance during the test conditions.

In our study, only adolescents could participate, as Path Control and FreeD are only available for the adult Lokomat exoskeleton. As soon as a pediatric version is available, the study should be extended to children as well. Furthermore, for safety reasons, the patients had to wear foot lifters while walking in the Lokomat and it is possible that this has influenced muscular activity, especially of the distal muscles.

Another limitation is that the treadmill speed in our patients varied between $1.8-2.2 \mathrm{~km} / \mathrm{h}$ to allow for comfortable walking. Since gait speed has an influence on the gait pattern [61], treadmill speed was kept constant throughout the whole experiment. Therefore, future strategies to increase patients' interactivity with robot devices should combine the tested modes of this study with approaches to adapt the treadmill speed according to the patient's intention [26,62]. The results of FreeD in this study were against our expectations, and further research is necessary to clarify this topic. We assume that the chosen settings of the control modes might have overly affected the difficulties of the walking conditions (e.g. Guidance Force set to $0 \%$ and Support Force to $100 \%$ in Path Control and FreeD, see Fig. 3). This is supported by the fact that Guidance Force had to be adjusted to $10 \%$ in 3 patients to enable 2 min of constant walking. Nevertheless, the idea of the experiment was to select 3 conditions with differing kinematic variability. Further studies should test the FreeD in combination with a bigger underlying Guidance Force (e.g. above $60 \%$ ), which will most likely be used in a clinical setting. Additionally, future studies should investigate changes of gait pattern over time (e.g. over 15 min. Walking time with the same conditions) and alterations/adaptations of the gait pattern after several training sessions with new technologies (including studies of effectiveness).

\section{Conclusion}

With this study, we could show that alterations in muscle activity (amplitude and pattern) can occur when different control modes are used during training with the Lokomat. Therefore, it seems that patient-cooperative tools are able to address the main point of criticism against robotassisted gait training: the passivity of the patient. Additionally, especially with Path Control, patients can train walking in an active and physiological way. Further studies should clarify, why the FreeD as tested in this study seems to be less applicable for physiologic walking in moderately affected children and adolescents with neurological gait disorders and which requirements patients must meet to train physiologically also with FreeD. Furthermore, longitudinal studies should be performed to address the effectiveness and long-term effects of these new control modes. However, the therapist is still the most important factor for evaluating and influencing the performance during robot-assisted gait therapy. It is his/her responsibility to guide the training process and to choose an adequate training mode to reach patients' individual aims and goals of gait rehabilitation.

\section{Additional files}

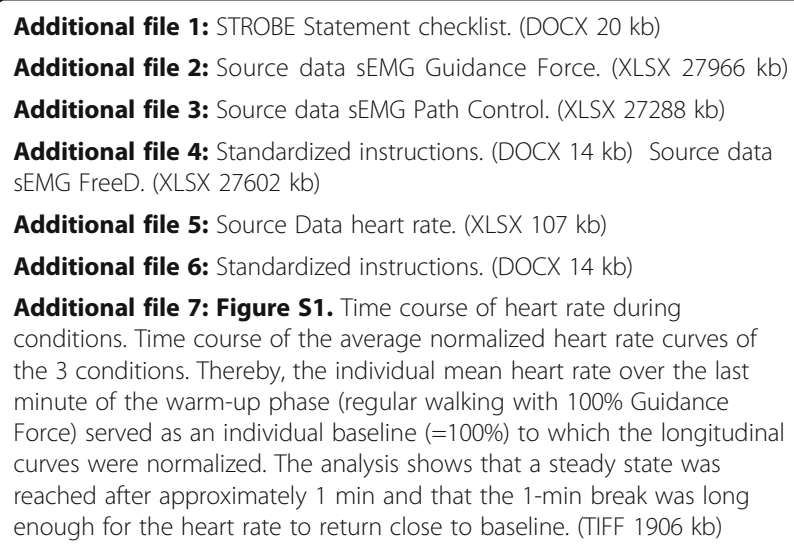

\section{Abbreviations}

BF: M.biceps femoris; FDR: False Discovery Rate; GL: M.gastrocnemius lateralis; GMFCS: Gross Motor Function Classification System; RF: M.rectus femoris; SD: Standard deviation; sEMG: Surface electromyography; TA: M.tibialis anterior; VM: M.vastus medialis

\section{Acknowledgements}

We thank the physiotherapist Sandra Ricklin (Rehabilitation Center Affoltern am Albis, Switzerland), who made an important contribution to the data acquisition, and Alexander Duschau-Wicke (Hocoma AG, Switzerland) who provided technical support with the new hardware and software modes. A special thanks goes to all patients and their parents for participating in the study. We further acknowledge the Neuroscience Center Zurich (ZNZ). 


\section{Funding}

This research was financially supported by the "Mäxi" Foundation, the Fondation Gaydoul, and the CRPP Neurorehabilitation of the Medical Faculty of the University of Zurich, Switzerland.

\section{Availability of data and materials}

The datasets supporting the conclusions of this article are included within the article and its additional files.

\section{Authors' contributions}

TA: Research project conception and execution, data acquisition review and critique, manuscript writing. FG: Research project organization and execution, data acquisition, statistical analysis, writing of the first draft. $\mathrm{HvH}$ : Research project conception, statistical analysis review, manuscript review. RL: Research project conception, statistical analysis, manuscript review and critique. All authors approved the final manuscript.

\section{Ethics approval and consent to participate}

This study was approved by the Cantonal Ethics Committee of Zurich (reference number KEK-ZH-Nr. StV. 07/07). All participants $\geq 14$ years and al legal guardians gave written informed consent to participate, participants $<14$ years provided assent.

\section{Consent for publication}

All authors have approved the manuscript for submission. All participants and or their legal guardians gave written informed consent to publish these data.

\section{Competing interests}

The authors declare that they have no competing interests and there are no financial competing interests to declare in relation to this manuscript.

\section{Publisher's Note}

Springer Nature remains neutral with regard to jurisdictional claims in published maps and institutional affiliations.

\section{Author details}

'Rehabilitation Center Affoltern am Albis, Children's University Hospital Zurich, Muehlebergstrasse 104, CH-8910 Affoltern am Albis, Switzerland. ${ }^{2}$ Children's Research Center, Children's University Hospital Zurich, Steinwiesstrasse 75, CH-8032 Zurich, Switzerland. ${ }^{3}$ Department of Health Sciences and Technology, ETH Zurich, Vladimir-Prelog-Weg 1-5/10, CH-8093 Zürich, Switzerland.

Received: 19 October 2016 Accepted: 28 June 2017

Published online: 14 July 2017

\section{References}

1. Dundar U, Toktas H, Solak O, Ulasli AM, Eroglu S. A comparative study of conventional physiotherapy versus robotic training combined with physiotherapy in patients with stroke. Top Stroke Rehabil. 2015;21(6):453-61.

2. Esclarín-Ruz A, Alcobendas-Maestro M, Casado-Lopez R, Perez-Mateos G, Florido-Sanchez MA, Gonzalez-Valdizan E, et al. A comparison of robotic walking therapy and conventional walking therapy in individuals with upper versus lower motor neuron lesions: a randomized controlled trial. Arch Phys Med Rehabil. 2014;95(6):1023-31.

3. Mehrholz J, Elsner B, Werner C, Kugler J, Pohl M. Electromechanical-assisted training for walking after stroke: updated evidence. Stroke. 2013;44(10):e127-8.

4. Beretta E, Romei M, Molteni E, Avantaggiato P, Strazzer S. Combined robotic-aided gait training and physical therapy improve functional abilities and hip kinematics during gait in children and adolescents with acquired brain injury. Brain Inj. 2015;29(7-8):955-62.

5. Schroeder AS, Homburg M, Warken B, Auffermann H, Koerte I, Berweck S, et al. Prospective controlled cohort study to evaluate changes of function, activity and participation in patients with bilateral spastic cerebral palsy after robot-enhanced repetitive treadmill therapy. Eur J Paediatr Neurol. 2014;18(4):502-10

6. Mayr A, Kofler M, Quirbach E, Matzak H, Fröhlich K, Saltuari L. Prospective, blinded, randomized crossover study of gait rehabilitation in stroke patients using the Lokomat gait orthosis. Neurorehabil Neural Repair. 2007;21(4): 307-14.
7. Schwartz I, Sajin A, Fisher I, Neeb M, Shochina M, Katz-Leurer M, et al. The effectiveness of locomotor therapy using robotic-assisted gait training in subacute stroke patients: a randomized controlled trial. PM R. 2009; 1(6):516-23.

8. Hidler J, Hamm LF, Lichy A, Groah SL. Automating activity-based interventions: the role of robotics. J Rehabil Res Dev. 2008;45(2):337-44.

9. Hornby TG, Campbell DD, Kahn JH, Demott T, Moore JL, Roth HR. Enhanced gait-related improvements after therapist- versus robotic-assisted locomotor training in subjects with chronic stroke: a randomized controlled study. Stroke. 2008;39(6):1786-92

10. Dobkin BH, Duncan PW. Should body weight-supported treadmill training and robotic-assistive steppers for locomotor training trot back to the starting gate? Neurorehabil Neural Repair. 2012 May;26(4):308-17.

11. Labruyère R, van Hedel HJA. Strength training versus robot-assisted gait training after incomplete spinal cord injury: a randomized pilot study in patients depending on walking assistance. J Neuroeng Rehabil. 2014;11(1):4.

12. Reinkensmeyer DJ, Emken $\mathrm{J}$, Cramer SC. Robotics, motor learning, and neurologic recovery. Annu Rev Biomed Eng. 2004 Jan;6:497-525.

13. Mirelman A, Bonato $P$, Deutsch JE. Effects of training with a robot-virtual reality system compared with a robot alone on the gait of individuals after stroke. Stroke. 2009:40(1):169-74.

14. Krishnan C, Ranganathan R, Dhaher YY, Rymer WZ. A pilot study on the feasibility of robot-aided leg motor training to facilitate active participation. PLoS One. 2013:8:10

15. Hidler JM, Wall AE. Alterations in muscle activation patterns during roboticassisted walking. Clin Biomech. 2005;20(2):184-93.

16. Israel JF, Campbell DD, Kahn JH, Hornby TG. Metabolic costs and muscle activity patterns during robotic- and therapist-assisted treadmill walking in individuals with incomplete spinal cord injury. Phys Ther. 2006;86(11):1466-78.

17. Lotze M, Braun C, Birbaumer N, Anders S, Cohen LG. Motor learning elicited by voluntary drive. Brain. 2003;126(4):866-72.

18. Kaelin-Lang A, Sawaki L, Cohen LG. Role of voluntary drive in encoding an elementary motor memory. J Neurophysiol. 2005;93(2):1099-103.

19. Reinkensmeyer DJ, Akoner OM, Ferris DP, Gordon KE. Slacking by the human motor system: Computational models and implications for robotic orthoses. Proceedings of the Annual International Conference of the IEEE Engineering in Medicine and Biology Society. 2009;2129-32.

20. Lewek MD, Cruz TH, Moore JL, Roth HR, Dhaher YY, Hornby TG. Allowing Intralimb kinematic variability during Locomotor training Poststroke improves kinematic consistency: a subgroup analysis from a randomized clinical trial. Phys Ther. 2009;89(8):829-39.

21. Bernstein NA. The co-ordination and regulation of movements. Oxford: Pergamon Press; 1967

22. Cai LL, Fong AJ, Otoshi CK, Liang YQ, Cham JG, Zhong H, et al. Effects of consistency vs. variability in robotically controlled training of stepping in adult spinal mice. IEEE 9th International Conference on rehabilitation robotics; 2005. p. 575-9.

23. Marchal-Crespo L, Reinkensmeyer DJ. Review of control strategies for robotic movement training after neurologic injury. J Neuroeng Rehabil. 2009;6(1):20

24. Colombo G, Joerg M, Schreier R, Dietz V. Treadmill training of paraplegic patients using a robotic orthosis. J Rehabil Res Dev. 2000; 37(6):693-700.

25. Riener R, Lünenburger $L$, Jezernik S, Anderschitz M, Colombo G, Dietz V. Patient-cooperative strategies for robot-aided treadmill training: first experimental results. IEEE Trans Neural Syst Rehabil Eng. 2005;13(3):380-94.

26. Duschau-Wicke A, von Zitzewitz J, Caprez A, Lunenburger L, Riener R. Path control: a method for patient-cooperative robot-aided gait rehabilitation. IEEE Trans Neural Syst Rehabil Eng. 2010;18(1):38-48.

27. Schück $A$, Labruyère $R$, Vallery $H$, Riener $R$, Duschau-Wicke A. Feasibility and effects of patient-cooperative robot-aided gait training applied in a 4-week pilot trial. J Neuroeng Rehabil. 2012;9(1):31.

28. Cai LL, Fong AJ, Otoshi CK, Liang Y, Burdick JW, Roy RR, et al. Implications of assist-as-needed robotic step training after a complete spinal cord injury on intrinsic strategies of motor learning. J Neurosci. 2006;26(41):10564-8.

29. Banala SK, Kim SH, Agrawal SK, Scholz JP. Robot assisted gait training with active leg exoskeleton (ALEX). IEEE Trans neural Syst Rehabil Eng. 2009;17(1):2-8.

30. Duschau-Wicke A, Caprez A, Riener R. Patient-cooperative control increases active participation of individuals with $\mathrm{SCl}$ during robot-aided gait training. J Neuroeng Rehabil. 2010;7:43. 
31. Duschau-Wicke A, Felsenstein S, Riener R. Adaptive body weight support controls human activity during robot-aided gait training. 2009 IEEE International Conference on rehabilitation robotics, ICORR 2009; 2009. p. 413-8.

32. Krishnan C, Kotsapouikis D, Dhaher $Y Y$, Rymer WZ. Reducing robotic guidance during robot-assisted gait training improves gait function: a case report on a stroke survivor. Arch Phys Med Rehabil. 2013;94(6):1202-6.

33. Koopman B, Meuleman JH, van Asseldonk EHF, van der Kooij H. Lateral balance control for robotic gait training. IEEE Int conf Rehabil robot. 2013.

34. Veneman JF, Menger J, van Asseldonk EHF, van der Helm FCT, van der Kooij $\mathrm{H}$. Fixating the pelvis in the horizontal plane affects gait characteristics. Gait Posture. 2008;28(1):157-63.

35. Zutter D, Hamann SL, Bichler L. Technischer Fortschritt in der Gangtherapie : Rehaklinik Zihlschlacht und Hocoma bringen weltweit neues Lokomat Modul zum klinischen Einsatz. http://www.hocoma.com. Accessibility lastly checked 13 Mar 2014.

36. Liao HF, Jeng SF, Lai JS, Cheng CK, Hu MH. The relation between standing balance and walking function in children with spastic diplegic cerebral palsy. Dev Med Child Neurol. 1997;39(2):106-12.

37. Hocoma AG. Legal notes. http://www.hocoma.com. Accessibility lastly checked 13 March 2014.

38. Palisano R, Rosenbaum P, Walter S, Russell D, Wood E, Galuppi B. Development and reliability of a system to classify gross motor function in children with cerebral palsy. Dev Med Child Neurol. 1997;39(4):214-23.

39. Hislop H, Brown M. Daniels and Worthingham's muscle testing. 9th Edition. Philadelphia: WB Saunders Co; 2013.

40. Fowler EG, Staudt LA, Greenberg MB, Oppenheim WL. Selective control assessment of the lower extremity (SCALE): development, validation, and interrater reliability of a clinical tool for patients with cerebral palsy. Dev Med Child Neurol. 2009;51:607-14.

41. Colombo G, Wirz M, Dietz V. Driven gait orthosis for improvement of locomotor training in paraplegic patients. Spinal Cord. 2001;3:252-5.

42. Riener R, Lünenburger L, Maier I, Colombo G, Dietz V. Locomotor training in subjects with sensori-motor deficits: an overview of the robotic gait orthosis Lokomat. J Healthc Eng. 2010;1(2):216.

43. Aurich-Schuler T, Warken B, Graser J V, Ulrich T, Borggraefe I, Heinen F, et al. Practical recommendations for robot-assisted treadmill therapy (Lokomat) in children with cerebral palsy: Indications, goal setting, and clinical implementation within the WHO-ICF framework. Neuropediatrics. 2015;46(4): 248-60

44. Urbaniak GC, Plous S. Research Randomizer V4.0. 2013. Available from: http://www.randomizer.org/. Accessibility lastly checked 11 Oct 2016.

45. World Medical Association. World Medical association declaration of Helsinki: ethical principles for Medical research involving human subjects. Bulletin of the World Health Organization. 2001;79(4):373-4.

46. Hermens HJ, Freriks B, Disselhorst-Klug C, Rau G. Development of recommendations for SEMG sensors and sensor placement procedures. J Electromyogr Kinesiol. 2000;10(5):361-74.

47. Shiavi R, Frigo C, Pedotti A. Electromyographic signals during gait: criteria for envelope filtering and number of strides. Med Biol Eng Comput. 1998; 36(2):171-8

48. Chang W-N, Lipton JS, Tsirikos Al, Miller F. Kinesiological surface electromyography in normal children: range of normal activity and pattern analysis. J Electromyogr Kinesiol. 2007:17(4):437-45.

49. Rohatgi A. WebPlotDigitizer 3.10. Austin, Texas, USA. Available from: http:// arohatgi.info/WebPlotDigitizer. Accessibility lastly checked 11 Oct 2016

50. Benjamini Y, Hochberg Y. Controlling the False Discovery rate: a practica and powerful approach to multiple testing. J R Stat Soc Ser B Methodol. 1995;57(1):289-300.

51. Cohen J. Statistical Power Analysis for the Behavioral Sciences. 2nd ed. Erlbaum L \& A, editor. Routledge; 1988.

52. Evans JD. Straightforward statistics for the Behavioral Sciences. Brooks/Cole pub. Pacific Grove: Co: An International Thomsom Publ. Co; 1996.

53. van Kammen K, Boonstra AM, van der Woude LHV, Reinders-Messelink HA, den Otter R. The combined effects of guidance force, bodyweight support and gait speed on muscle activity during able-bodied walking in the Lokomat. Clin Biomech. 2016:36:65-73.

54. Duschau-Wicke AHB. Patient-cooperative robot-aided gait rehabilitation. DISS. ETH NO. 19300. Zurich, Switzerland; 2010.

55. Agostini V, Nascimbeni A, Gaffuri A, Imazio P, Benedetti MG, Knaflitz M, et al. Gait \& Posture Normative EMG activation patterns of school-age children during gait. Gait Posture. 2010;32(3):285-9.
56. Aurich-Schuler T, Müller R, van Hedel HJA. Leg surface electromyography patterns in children with neuro-orthopedic disorders walking on a treadmill unassisted and assisted by a robot with and without encouragement. J Neuroeng Rehabil. 2013;10:78.

57. Brunner R, Romkes J. Abnormal EMG muscle activity during gait in patients without neurological disorders. Gait Posture. 2008;27(3):399-407.

58. Moreno JC, Barroso F, Farina D, Gizzi L, Santos C, Molinari M, et al. Effects of robotic guidance on the coordination of locomotion. J Neuroeng Rehabil. 2013;10:79.

59. Aoyagi D, Ichinose WE, Harkema SJ, Reinkensmeyer DJ, Bobrow JE. A robot and control algorithm that can synchronously assist in naturalistic motion during body-weight-supported gait training following neurologic injury. IEEE Trans neural Syst Rehabil Eng. 2007;15(3):387-400.

60. Huang VS, Krakauer JW. Robotic neurorehabilitation: a computational motor learning perspective. J Neuroeng Rehabil. 2009:6:5.

61. van Hedel HJA, Tomatis L, Müller R. Modulation of leg muscle activity and gait kinematics by walking speed and bodyweight unloading. Gait Posture. 2006;24(1):35-45.

62. von Zitzewitz J, Bernhardt M, Riener R. A novel method for automatic treadmill speed adaptation. IEEE Trans neural Syst Rehabil Eng. 2007;15(3):401-9.

\section{Submit your next manuscript to BioMed Central and we will help you at every step:}

- We accept pre-submission inquiries

- Our selector tool helps you to find the most relevant journal

- We provide round the clock customer support

- Convenient online submission

- Thorough peer review

- Inclusion in PubMed and all major indexing services

- Maximum visibility for your research

Submit your manuscript at www.biomedcentral.com/submit
) Biomed Central 